\title{
DIELECTRIC BEHAVIOUR OF POTASSIUM ACETYLACETONATE
}

\author{
T.G. Abdel-MaliK ${ }^{a}$, M.E. Kassem ${ }^{b}$, R.M. Abdel-Latif ${ }^{a}$ \\ AND S.M. KHALIL ${ }^{b}$ \\ a Department of Physics, Faculty of Science, El-Minia University, Egypt \\ ${ }^{b}$ Department of Physics, Faculty of Science, Alexandria University, Alexandria, Egypt
}

(Received February 18, 1992; revised version April 3, 1992)

\begin{abstract}
The dielectric constant and the dielectric loss of potassium acetylacetonate, $K(\mathbf{a c a c})$, are measured as a function of both temperature and frequency. A peak value is obtained at the a critical temperature $T_{c}=313 \mathrm{~K}$. X-ray analysis revealed that at $T_{\mathrm{c}}, \mathrm{K}(\mathrm{acac})$ undergoes phase transition from orthorhombic to triclinic structure. The Cole-Cole diagrams have been used to determine the molecular relaxation time $\tau$. The temperature dependence of $\tau$ is expressed by thermally activated process. The impedance spectra in the frequency range from $50 \mathrm{~Hz}$ to $500 \mathrm{kHz}$ plotted in the complex plane show semicircles. The conduction mechanism, below $T_{c}$, is predominantly operating at the surface. Above $T_{c}$, the conduction is proceeding in the bulk material with contribution from the surface.
\end{abstract}

PACS numbers: $77.40 .+i$

\section{Introduction}

The electrical properties of metal-acetylacetonate have attracted interest during the last few decades, because they exhibit ferroelectric behaviour. The dielectric properties of sodium acetylacetonate were studied [1]. A peak value in the dielectric constant was observed and has been ascribed to some structural irreversible change from orthorhombic phase to triclinic structure. The dielectric properties of potassium acetylacetonate $\mathrm{K}(\mathrm{acac})$ were also studied [2]. Two peaks were observed in the dielectric constant versus temperature curve at 283 and $294 \mathrm{~K}$, which were explained as irreversible transition temperatures. As far as we are aware, the structure of $\mathrm{K}$ (acac) has not been previously reported in the literature. The determination of such a structure, below room temperature, is not available. Therefore, we carried out our investigations above room temperature. Despite a considerable effort of understanding the dielectric properties of these compounds, the problem is still far from being complete. The aim of this work is to study the dielectric properties of $K(\mathrm{acac})$ as a function of both temperature and frequency. 
In addition, $\mathrm{X}$-ray diffraction pattern is made to give some knowledge about the structure of this compound.

\section{Experimental procedure}

Potassium acetylacetonate $\mathrm{K}(\mathrm{acac})$ was prepared according to standard procedures given in the literature [3]. The samples in the pellets form (diameter $10 \mathrm{~mm}$; thickness $2-4 \mathrm{~mm}$ ) were obtained by compressing the powder in a die under the pressure of $9.8 \times 10^{8} \mathrm{~Pa}$. Silver paste was painted on both flat surfaces of the pellet as electrodes. The dielectric constant, dielectric loss and impedance were measured at different temperatures (293 to $343 \mathrm{~K}$ ) and at various frequencies $(50$ to $500 \mathrm{kHz})$ using a Tesla BM 507 impedance meter.

\section{Results and discussion}

Figures $1 \mathrm{a}$ and $1 \mathrm{~b}$ show the temperature dependence of the dielectric constant $\varepsilon^{\prime}(\nu)$ and the dielectric loss $\varepsilon^{\prime \prime}(\nu)$ of $\mathrm{K}(\mathrm{acac})$, where $\nu$ is the frequency. As it can be seen from Fig. 1a, the dielectric constant shows a maximum at the critical temperature $T_{\mathrm{c}}=313 \mathrm{~K}$. The observed behaviour is similar to that obtained in
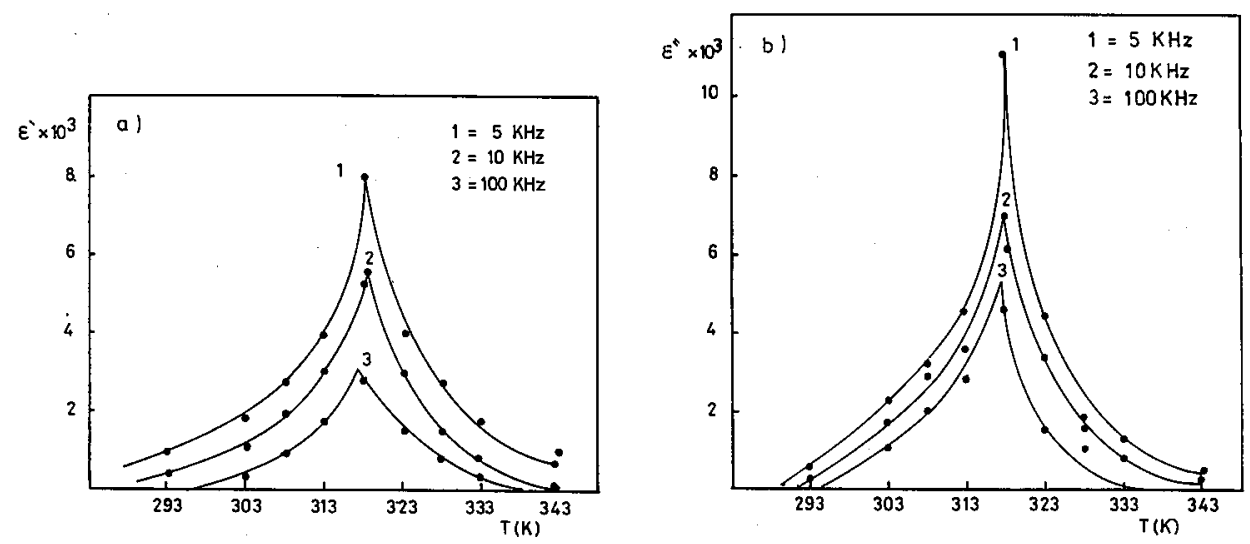

Fig. 1. (a) Dielectric constant as a function of temperature for $K$ (acac) at different frequencies. (b) Dielectric loss as a function of temperature for $K$ (acac) at different frequencies.

ferroelectric materials [4]. The Curie-Weiss constants evaluated from the slopes of the temperature dependence of $1 / \varepsilon^{\prime}$ at $5 \mathrm{kHz}$ were found to be $(2.6 \pm 0.1) \times 10^{4} \mathrm{~K}$ and $(2.0 \pm 0.1) \times 10^{4} \mathrm{~K}$ below and above $T_{\mathrm{c}}$, respectively.

$\mathrm{X}$-ray diffraction patterns below and above $T_{\mathrm{c}}$ were thus made on the $\mathrm{K}$ (acac) sample. The recorded charts of X-ray patterns are shown in Fig. 2. Ito's method [5] was used to index the powder X-ray diffraction patterns. It was found that the unheated $\mathrm{K}$ (acac) compound belongs to orthorhombic system whose unit cell has the following dimensions: 

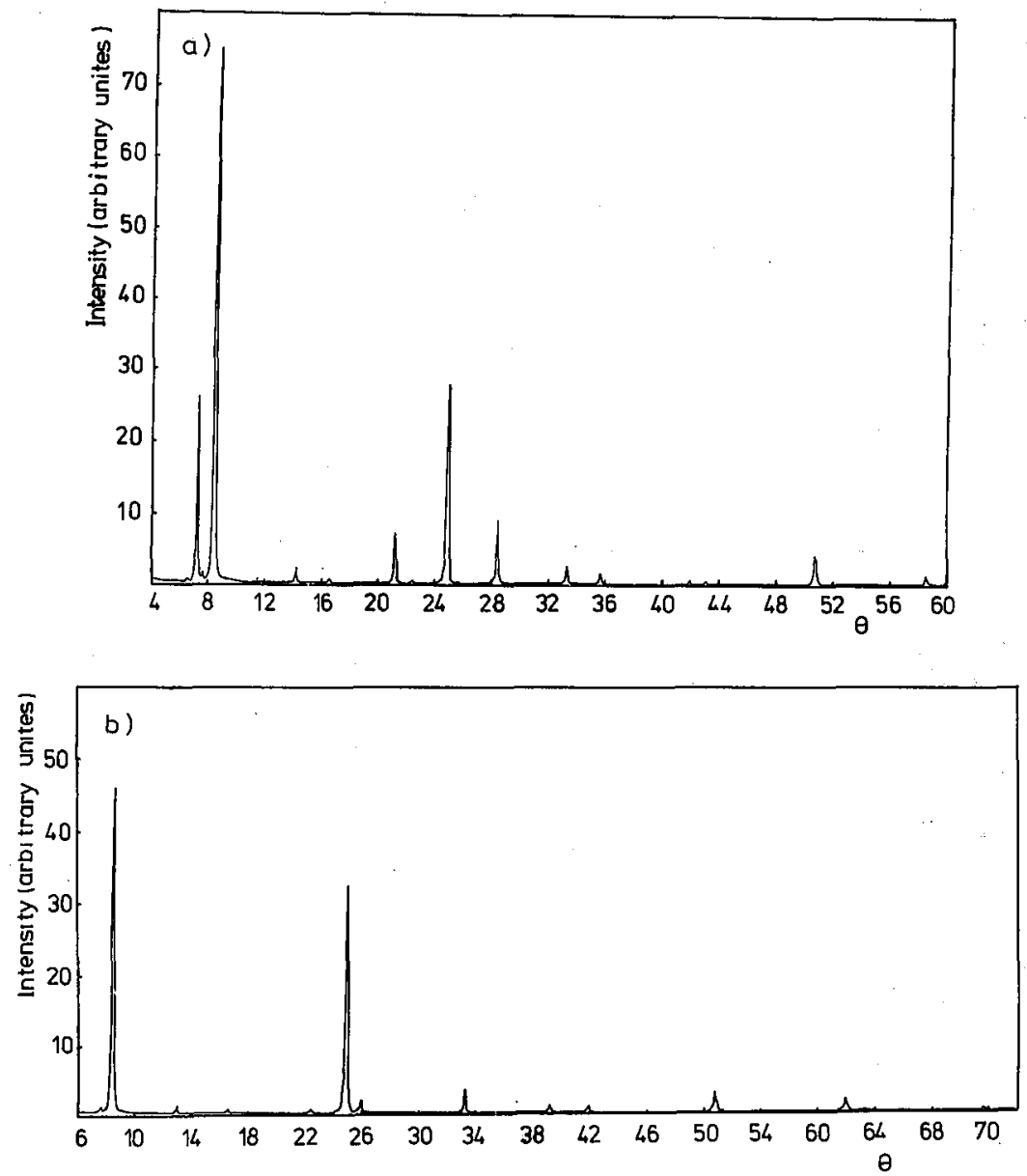

Fig. 2. X-ray diffraction pattern for $K\left(\right.$ acac): (a) below $T_{c} ;(b)$ above $T_{c}$.

$$
\begin{array}{ll}
a=13.36 \AA, & \alpha=90^{\circ}, \\
b=12.60 \AA, & \beta=90^{\circ}, \\
c=11.62 \AA, & \gamma=90^{\circ} .
\end{array}
$$

On the other hand, it was found that the heated compound (for $T>T_{\mathrm{c}}$ ) belongs to triclinic system whose unit cell dimensions are

$$
\begin{array}{ll}
a=11.9 \AA, & \alpha=108^{\circ} 52^{\prime}, \\
b=11.2 \AA, & \beta=89^{\circ} 12^{\prime}, \\
c=9.36 \AA, & \gamma=99^{\circ} 55^{\prime} .
\end{array}
$$

Therefore, at the transition temperature $T_{\mathrm{c}} \mathrm{K}$ (acac) transforms from orthorhombic to triclinic system.

The Cole-Cole diagrams $\varepsilon^{\prime \prime}\left(\varepsilon^{\prime}\right)$ in $K($ acac) are shown at several temperatures in Fig. 3. These diagrams have been used to determine the distribution parameter 
$\alpha$, the macroscopic relaxation time $\tau_{0}$, and the molecular relaxation time $\tau[6,7]$. Knowing $\alpha$, one can determine $\tau_{0}$ using the relation [6]

$$
\frac{U}{V} \doteq\left(\omega \tau_{0}\right)^{1-\alpha},
$$

where $U$ is the distance on the Cole-Cole diagram between the static dielectric constant $\varepsilon_{0}$ and the experimental point, $V$ is the distance between that point and the optical dielectric constant $\varepsilon_{\infty}$, and $\omega$ is the angular frequency, $2 \pi \nu$. The symbols $\varepsilon_{0}, \varepsilon_{\infty}, \alpha, U$ and $V$ are shown in Fig. 3 .
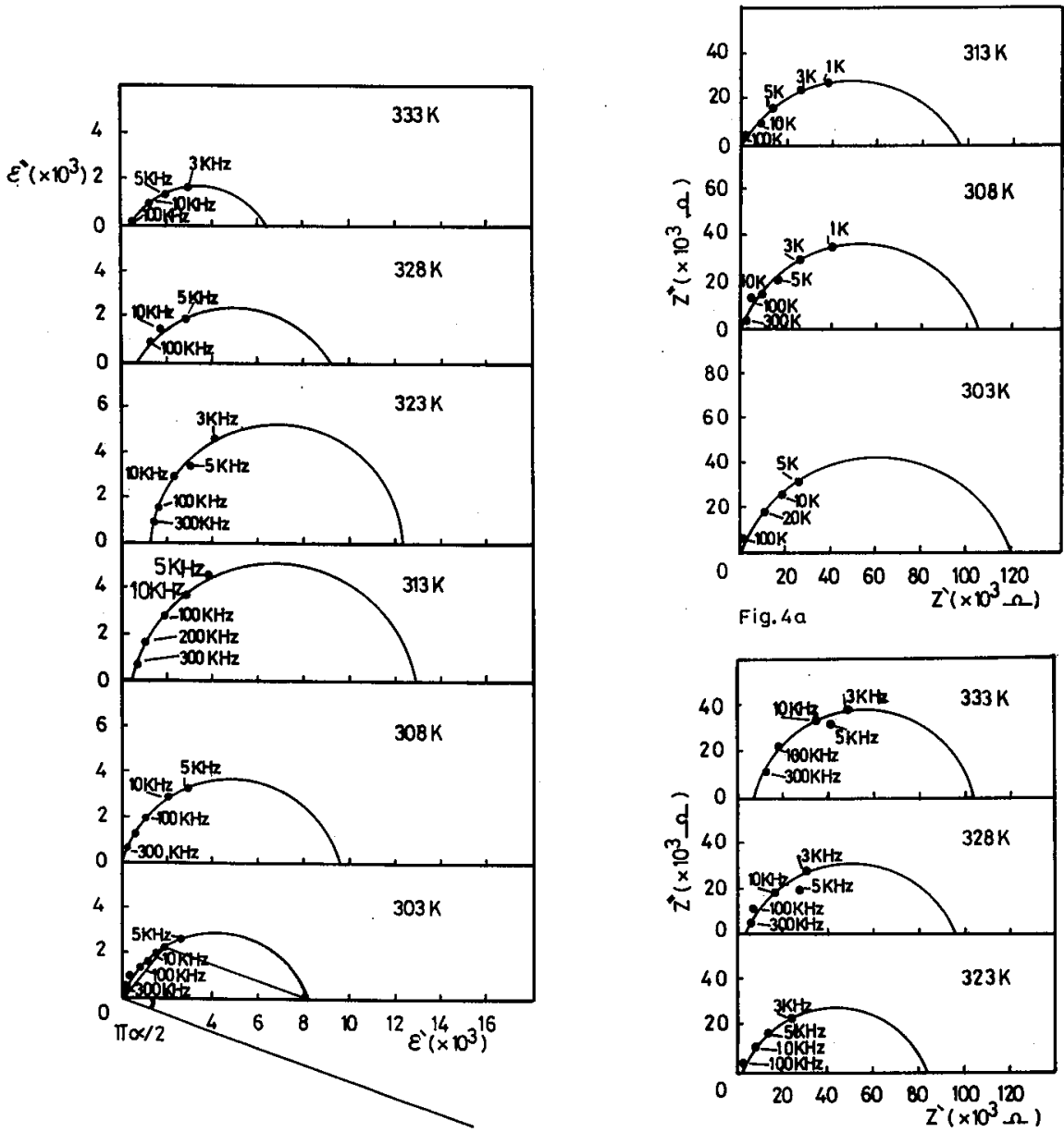

Fig. 3. The Cole-Cole diagram in $\mathrm{K}(\mathrm{acac})$ for a range of temperatures. The frequencies reported are in hertz.

Fig. 4. Complex impedance spectra of $K$ (acac) for a range of temperatures: (a) below $T_{c}$; (b) above $T_{c}$. The frequencies indicated are in hertz. 
The molecular relaxation time $\tau$ could be evaluated from

$\tau=\frac{2 \varepsilon_{0}+\varepsilon_{\infty}}{3 \varepsilon_{0}} \tau_{0}$

The temperature dependence of $\tau$ is controlled by the following relation [8]:

$\tau=A \exp ^{-E_{0} / k T}$,

where $A$ is constant, $k$ is Boltzmann's constant and $E_{0}$ is the activation energy for relaxation.

The calculated values of $E_{0}$ and $A$ were found to be $E_{0}=0.08 \pm 0.01 \mathrm{eV}$ and $A=(4.2 \pm 0.2) \times 10^{-3} \mathrm{~s}$ below $T_{\mathrm{c}}$ and $E_{0}=0.30 \pm 0.01 \mathrm{eV}$ and $A=(8.1 \pm$ $0.2) \times 10^{-9} \mathrm{~s}$ above $T_{\mathrm{c}}$, respectively.

The measured impedance $Z(\nu)=Z^{\prime}(\nu)+\mathrm{i} Z^{\prime \prime}(\nu)$ were plotted in the complex plane for various temperatures. Typical spectra related to the frequency $\nu$ of the applied sinusoidal voltage are shown in Fig. 4.

The frequency dependence of both $Z^{\prime}$ and $Z^{\prime \prime}$ permits separation of the sample resistance for the grain bulk $R_{\mathrm{b}}$ from the surface $R_{\mathrm{s}}$ [9]. The classical semicircular form shown in Fig. 4 leads to an equivalent circuit for $\mathrm{K}(\mathrm{acac})$ comprising $R_{\mathrm{b}}$ in series with parallel $R_{\mathrm{s}}-C_{\mathrm{s}}$ combination; this leads to the established relations

$$
\begin{aligned}
& Z^{\prime}=R_{\mathrm{b}}+R_{\mathrm{s}} /\left[1+\left(\omega C_{\mathrm{s}} R_{\mathrm{s}}\right)^{2}\right], \\
& Z^{\prime \prime}=\mathrm{i} \omega C_{\mathrm{s}} R_{\mathrm{s}}^{2} /\left[1+\left(\omega C_{\mathrm{s}} R_{\mathrm{s}}\right)^{2}\right] .
\end{aligned}
$$

It is clear that $Z^{\prime}$ varies throughout the applied frequency and is equal to $\left(R_{\mathrm{b}}+R_{\mathrm{s}}\right)$ at zero frequency (DC) and only $R_{\mathrm{b}}$ at higher frequency. Therefore, the complex semicircle $Z^{\prime \prime}\left(Z^{\prime}\right)$ impedance diagram would reflect $R_{\mathrm{b}}$ at higher frequencies whereas contribution of $R_{\mathrm{s}}$ increases upon shifting to the zero frequency (DC). It can be seen from Fig. 4 that below $T_{\mathrm{c}}$ the intersection of the real axis with zero frequency extrapolation of the semicircle gives $R_{\mathrm{s}}$, the surface resistance of the sample. The activation energy of the surface corduction was found to be $0.17 \pm 0.01 \mathrm{eV}$. Therefore, it is believed that the conduction is of ionic character predominantly operating at the surface. Above $T_{\mathrm{c}}$, the semicircle $Z^{\prime \prime}\left(Z^{\prime}\right)$ dependence would suggest conduction via ionic mechanism proceeding in the bulk material with contribution from the surface. The activation energies for the bulk and surface conduction were found to be $0.78 \pm 0.01 \mathrm{eV}$ and $0.17 \pm 0.01 \mathrm{eV}$, respectively.

\section{Conclusion}

Dielectric measurements reported for $K(\mathrm{acac})$ reveal the following items:

1. The anomalous behaviour in the dielectric properties of $\mathrm{K}(\mathrm{acac})$ is attributed to the structural phase transition from orthorhombic to triclinic system.

2. The molecular relaxation time $\tau$, determined from the Cole-Cole diagrams, reveals its activated character.

3. Ionic conduction operating at the surface is exhibited below $T_{c}$, above which the conduction is via ionic mechanism proceeding in the bulk material with contribution from the surface. 


\section{References}

[1] R. Kamel, M. Hilal, A.H. Eid, A. Sawaby, Mol. Cryst. Liq. Cryst. 31, 9 (1975).

[2] N.M. Molokhia, F. El-Shahat, J. Chem. Tech. Biotechnol. 31, 1 (1981).

[3] A.I. Vogel, Text-Book of Practical Organic Chemistry, 3rd ed., Longmans, London 1956, p. 865.

[4] A.K. Jonscher, Dielectric Relaxation in Solids, Chelsea Dielectrics Press Ltd., London 1983.

[5] T. Ito, X-ray Studies on Polymorphism, Maruzen Co. Ltd., Tokyo 1950.

[6] R.H. Cole, K.S. Cole, J. Chem. Phys. 9, 314 (1941).

[7] K.K. Srivastava, A. Kumaro, O.S. Panwar, K.MN. Lakshminarayan, J. Non-Cryst. Solids 33, 205 (1979).

[8] V.V. Danial, Dielectric Relaxation, Academic Press, London, New York 1967.

[9] J. Millete, M. Gillou, J. Chem. Phys. 64, 1726 (1967). 